American Journal of Pharmacology and Toxicology 2 (2): 30-38, 2007

ISSN $1557-4962$

(C) 2006 Science Publications

\title{
CPI-1189 Protects against TNBS-induced Colitis in a Rodent Model
}

\author{
Beverley Greenwood-Van Meerveld and Karl Tyler \\ VA Medical Center and Oklahoma Center for Neuroscience \\ University of Oklahoma Health Sciences Center, Oklahoma City, OK 73104
}

\begin{abstract}
Nitrone-related therapeutics (NRTs) represents a new class of molecules that may be effective in treating inflammatory conditions. The goal of this study was to examine the effect of CPI1189 in a rat model of inflammatory bowel disease (IBD). Colonic inflammation was induced by an enema of trinitrobenzene sulfonic acid (TNBS). Saline-enema treated rats served as controls. After 3 days colonic damage was assessed morphologically using a validated scoring system and through measurement of myeloperoxidase (MPO), an enzymatic marker of inflammation. We found that a dosedependent protection from TNBS-induced colonic damage was observed in rats given 10-70 mg kg-1 of CPI-1189 treated p.o. $1 \mathrm{hr}$. before the TNBS enema. Consistent with the 55\% absolute oral bioavailability of CPI-1189, greater protection (77\%) was seen when CPI-1189 $\left(30 \mathrm{mg} \mathrm{kg}^{-1}\right)$ was administered intravenously $1 \mathrm{hr}$. before the TNBS enema. In a separate series of healing experiments where CPI-1189 was administer after the TNBS inflammatory response had peaked, CPI-1189 at a dose of $90 \mathrm{mg} \mathrm{kg}^{-1}$ p.o. reduced the TNBS colitis. CPI-1189 prevents and heals TNBS-induced colonic damage in rats suggesting that CPI-1189 may be a novel agent for the treatment of IBD.
\end{abstract}

Key words: Inflammatory bowel disease, trinitrobenzene sulfonic acid, colon, rat

\section{INTRODUCTION}

Inflammatory bowel disease (IBD) is a blanket term for either Crohn's disease or ulcerative colitis. Clinically, IBD is characterized by bloody diarrhea and abdominal pain that is often accompanied by fever, weight loss, anemia and severe fatigue. Individuals with IBD also have an increased risk of developing colon cancer compared to the general population ${ }^{[1]}$. The cause of IBD remains unknown, although several factors such as genetic, infectious and immunologic susceptibility have been implicated ${ }^{[2]}$. Treatment options for patients with either Crohn's disease or colitis are limited. The paucity of a safe and effective pharmacological agent to treat IBD has led to the use of surgery to treat many severe cases of IBD. The poor side-effect profile of corticosteroids limits their use in patients with IBD to the treatment of acute flares. Products based on 5-aminosalicylic acid (5-ASA) as the active agent show frequent drug intolerance, limited efficacy and can only be used in mild to moderate cases. If corticosteroids and 5-ASA agents are ineffective or cannot be tolerated, powerful immunosuppressive agents with significant side-effects such as cyclosporine, 6-mecaptopurine or azathioprine are tried. Advances in the treatment of IBD have been difficult to achieve because of the lack of a full understanding of the etiology and pathophysiology of the disease. Monoclonal antibodies, such as the recently introduced antibody to TNF- $\alpha$, represent a new therapeutic advance in the treatment of patients with IBD. However, the long-term toxic effects of these potentially immunogenic proteins remain to be determined.

CPI-1189 (Fig. 1A) is representative of a new class of therapeutic agents related to $\alpha$-phenyl butyl nitrone (Fig. 1B), a compound that has been shown to mitigate oxidative stress in numerous disease models of ischemia and inflammation ${ }^{[3,4]}$. Oxidative stress is suggested to play a crucial role in $\operatorname{IBD}^{[5]}$ and the purpose of the present study was to investigate the efficacy and potency of CPI-1189 in a standard in vivo animal model of IBD, TNBS-induced colitis ${ }^{[6]}$.

\section{MATERIALS AND METHODS}

Animals: Male Sprague Dawley rats (B \& K Universal, Fremont, CA.) were used for these experiments. For the

Corresponding Author: Beverley Greenwood-Van Meerveld, Ph.D., FACG, VA Medical Center, Research Administration, Room 151, 921 NE 13th St., Oklahoma City, OK 73104 Tel: (405)-270-0501 (Ext. 3547) Fax: (405)-290-1719 
TNBS experiments the weight range of the animals was 200-412 g. Animals were maintained on standard rat chow and tap water ad libitum and were housed under controlled conditions of illumination (12 hr. light-dark cycle) and temperature $\left(22^{\circ} \mathrm{C}\right)$. Rats in the TNBS study were fasted for 18-24 hr. prior to experimentation and allowed food and water ad libitum after the treatment.

A.

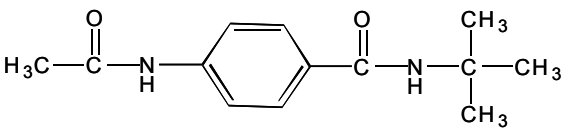

\section{CPI-1189}

B.

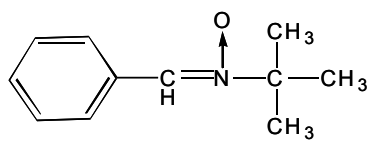

\section{PBN}

Fig. 1: Chemical structure of CPI-1189 (A) and PBN (B)

For the TNBS study rats were housed 2 per cage in the animal facility at the V.A. Research Center in Oklahoma. For the dose proportionality and absolute bioavailability studies rats were housed in the animal facility at Centaur Pharmaceuticals in Sunnyvale, CA for at least 7 days prior to experimentation. Until one day prior to dosing, the animals were housed 3 rats cage $^{-1}$; thereafter, the animals were singly housed. Animals in the dose proportionality study were fasted for $2 \mathrm{hr}$. prior to experimentation and allowed food and water ad libitum for $0.5 \mathrm{hr}$. after the treatment. Animals in the absolute bioavailability study were fasted for 2 $\mathrm{hr}$. prior to experimentation and allowed food and water ad libitum for $1 \mathrm{hr}$. after the treatment.

Induction of colonic inflammation in rats: Fasted animals were brought into the laboratory and colonic damage was induced by instillation of TNBS $(50 \mathrm{mg}$ $\mathrm{kg}^{-1}$ dissolved in $25 \%$ ethanol in a $0.5 \mathrm{~mL}$ enema) delivered directly into the colon through the anal opening via a $8 \mathrm{~cm}$ polyethylene tube attached to a $1 \mathrm{~mL}$ syringe.

Treatment protocol (TNBS experiments): CPI-1189 was suspended in 1\% methyl cellulose and then stirred overnight before dosing. In experiments designed to investigate the ability of CPI-1189 to prevent colonic damage induced by TNBS, animals were dosed orally with a $2 \mathrm{~mL}$ bolus of CPI-1189 (10, 30, or $\left.70 \mathrm{mg} \mathrm{kg}^{-\mathrm{r}}\right)$ or the methyl cellulose vehicle one hr. pre-TNBS instillation. In the experiment to investigate the healing capacity of CPI-1189, animals were dosed orally with the compound $\left(90 \mathrm{mg} \mathrm{kg} \mathrm{kg}^{-1}\right.$ ) or its methyl cellulose vehicle 4,8 or $24 \mathrm{hr}$ post-TNBS enema. To determine the efficacy of CPI-1189 following intravenous administration, a series of experiments were performed in which $30 \mathrm{mg} \mathrm{kg}^{-1}$ CPI-1189 (0.5 mL) was dosed via the tail-vein. Three days following the TNBS induction of colitis animals were euthanized and then a $5 \mathrm{~cm}$ segment of distal colon was removed, opened longitudinally and cleansed of fecal material. A longitudinal strip of the colonic segment weighing approximately $20-40 \mathrm{mg}$ was then isolated and placed into a cryo-storage vial and quick frozen in liquid $\mathrm{N}_{2}$ for later MPO analysis. The weight of the remaining colon was then recorded and the colon was placed on to a plastic viewing tray for gross morphological scoring.

\section{Assessment of colonic damage and inflammation}

Morphological scoring: The morphological score was determined by a qualified blinded observer. A 0-5 rating scale ${ }^{[6]}$ was assigned for each tissue sample examined; $0=$ no damage, $1=$ localized hyperemia but no ulcers, $2=$ ulcers with no significant inflammation, $3=$ ulcers with inflammation at one site; $4=$ two or more sites of inflammation and /or ulceration, $5=$ Two or more major sites of inflammation and/or ulceration, or one major site of inflammation and ulceration extending $>1 \mathrm{~cm}$ along the colon. For each treatment group, the morphological scores were combined and means \pm SE were calculated.

Myeloperoxidase (MPO) activity: MPO is an enzyme that is released into tissue under inflammatory conditions. Increases in MPO activity shows direct correlation to the severity of inflammatory damage ${ }^{[7]}$. MPO activity was measured using spectrophotometric analysis. Briefly, after determining the wet weight of the colonic tissue sample, it was homogenized in buffer (HTAB- $1 \mathrm{ml} / 20 \mathrm{ng}$ tissue) and then after a series of freeze -thaw steps, the homogenate was centrifuged for 2 min at $5000 \mathrm{RPM}$, then $10 \mu \mathrm{L}$ of the supernatant or a series of horse radish peroxidase (HRP) standards were placed into duplicate wells of ELISA microtiter plates. The peroxidase substrate (3,3', 5,5' tetramethylbenzidine- TMB) was added to each well to initiate an enzymatic reaction. After 10 minutes, the reaction was stopped by adding $100 \mu \mathrm{L}$ of $0.18 \mathrm{M}$ $\mathrm{H}_{2} \mathrm{SO}_{4}$. An ELISA reader was used to measure the optical density of converted substrate and MPO activity was quantified as $\mathrm{ng} / 100 \mu \mathrm{L}$ sample. These values were then expressed as MPO activity ng/g wet weight tissue.

Treatment protocol (dose-proportionality study): CPI-1189 was formulated as a suspension in 1\% aqueous methyl cellulose. Rats were dosed orally with CPI-1189 at 10,30 or $100 \mathrm{mg} \mathrm{kg}^{-1}$ (4 rats/dosing group). Dosing volumes were $0.005 \mathrm{~mL} \mathrm{~g}^{-1}$. Blood was collected, via the tail vein from each rat and placed into 
heparinized tubes, at $0,0.5,1,2,4,8,12,16,20$ and 24 hr. post-dose of CPI-1189.

Treatment protocol (absolute bioavailability study): CPI-1189 was administered $20 \mathrm{mg} \mathrm{kg}^{-1}$ either i.v. or by oral gavage. Four rats were dosed in each treatment group. The solution used for the i.v. treatment consisted of $75 \%$ polyethylene glycol (PEG) and $25 \%$ water. The suspension used for the p.o. treatment was prepared in $1 \%$ aqueous methylcellulose. Dosing volumes were $0.002 \mathrm{~mL} \mathrm{~g}^{-1}$ of body weight for both p.o. and i.v. treatments. Each rat dosed orally was sampled serially by tail bled into heparinized tubes pre-dose at $0.5,1,2$, 4, 8 and $24 \mathrm{hr}$. post-dose. Each rat dosed i.v. was sampled serially by tail bleed into heparinized tubes pre-dose and at $0.083,0.25,0.5,1,2,4,8$ and $24 \mathrm{hr}$. post-dose.

Analysis of CPI-1189 in rat blood: An aliquot of the whole rat blood from the tail bleeds was added to 0.5 $\mathrm{mL}$ methanol in a $1.5 \mathrm{~mL}$ microcentrifuge tube. The samples were centrifuged for 10 minutes at 14,000 rpm at $4^{\circ} \mathrm{C}$ and the supernatant was filtered into $800 \mu \mathrm{L}$ HPLC vials using a $0.45 \mu \mathrm{m}$ syringe filter and a $1 \mathrm{~mL}$ syringe. $50 \mu \mathrm{L}$ of this filtrate was injected onto the HPLC column. Using this procedure, the recovery of CPI-1189 was quantitative. The HPLC solvent mixture was $49.5 \% \quad 0.03 \mathrm{M}$ citrate/acetate buffer $(\mathrm{pH} 3.6)$ and $50.5 \%$ HPLC grade methanol. The flow rate was set to $1.0 \mathrm{~mL} \mathrm{~min}^{-1}$ and the run time was 10 minutes. The UV detector was set to $262 \mathrm{~nm}$. The CPI-1189 peak typically eluted between 6 and $7 \mathrm{~min}$. The absolute response from eluting CPI-1189 in an experimental sample was converted to a concentration using the slope $(\mathrm{m})$ and intercept (b) values from an nonweighted linear regression analysis of the response from the injection of comparable aliquots of methanol solutions representing CPI-1189 concentrations of 0 , 2.3, 4.7, 9.4, 19 and $23 \mu \mathrm{g} \mathrm{mL}^{-1}$. The lower and upper limits of quantification for CPI-1189 for this procedure were typically $2.3 \mu \mathrm{g} \mathrm{mL} \mathrm{mL}^{-1}$ and $94 \mu \mathrm{g} \mathrm{mL} \mathrm{m}^{-1}$, respectively, using $150 \mu \mathrm{L}$ of blood fluid for analysis. The assay had an inter-assay precision above $10 \mu \mathrm{g}$ $\mathrm{mL}^{-1}$ of $<10 \%$. Between 2.3 and $10 \mu \mathrm{g} \mathrm{mL}^{-1}$, the interassay precision was $<20 \%$.

Chemicals: $\quad$ CPI-1189 (4-acetylamino-N-tertbutylbenzamide) was synthesized and supplied by Centaur Pharmaceuticals, Sunnyvale CA. 2,4,6trinitrobenzenesulfonic acid (TNBS), horse radish peroxidase (HRP), methyl cellulose, hexadecyltrimethylammonium bromide, sodium phosphate, potassium phosphate, sulfuric acid and 3,3', 5,5 ' tetramethylbenzidine (TMB) were all supplied by SIGMA Chemical Co., St. Louis, MO.

\section{Data and statistical analysis}

Efficacy studies in TNBS rats: Values were expressed as mean \pm sem and data were analyzed using nonparametric tests in the SAS software package (SAS version 6.12, Cary, NC). Simple two-way comparisons were performed using PROC NPAR1WAY (Wilcoxon). Dose-response relationships were analyzed using ANOVA by rank (Proc Rank followed by analysis of the normalized values by PROC GLM). If the ANOVA by rank indicated a significant difference, group comparisons were performed with a Tukey posthoc test. Statistical significance was set as the $\mathrm{p}<0.05$ level and designated with an asterisk.

Dose proportionality and absolute bioavailability studies: The concentrations were tabulated and subjected to a noncompartmental pharmacokinetic analysis using WinNonlin (Professional Version 1.5, Scientific Consulting, Apex, NC) to determine the mean concentrations $\pm \mathrm{SD}$ and the pharmacokinetic parameters. Estimates for the AUC calculation the linear/log linear technique was used. Model 200 was used for p.o. dosing data and model 202 was used for the i.v. dosing data.

\section{RESULTS}

The intracolonic administration of TNBS (50 $\mathrm{mg}$ $\mathrm{kg}^{-1}$ in $25 \%$ ethanol) consistently produced colonic damage in the distal portion of the rat colon at 3 days post treatment. The gross morphological damage produced by TNBS was characterized by "patchy" areas of necrosis, ulceration, hyperemia and inflammation. After TNBS administration the macroscopic changes were accompanied by a 4fold increase in distal colonic MPO activity compared to that found in control rats not given the TNBS enema. The colonic damage induced by TNBS was also associated with a 4-fold increase in tissue wet weight.

\section{Protective effects of pretreatment with CPI-1189}

Efficacy of CPI-1189: In a our first series of experiments, groups of rats ( $\mathrm{n}=11 /$ group) were treated with either a single oral dose of CPI-1189 $\left(10 \mathrm{mg} \mathrm{kg}^{-1}\right)$ or the methylcellulose vehicle control $1 \mathrm{hr}$. before the TNBS enema. This dose of CPI-1189 was selected based on its ability to provide $50 \%$ neuroprotection in an in vivo model of (MPTP mouse model of Parkinson's disease - data on file Centaur Pharmaceuticals Inc.). Macroscopic analysis after 3 days revealed that at $10 \mathrm{mg} \mathrm{kg}^{-1}$ p.o. CPI-1189 had a significant protective effect $(46 \%)$ against the TNBSinduced morphological damage. In the vehicle-treated group $(\mathrm{n}=11)$ the morphological score was $2.4 \pm 0.4$ whereas in the CPI-1189 treated group there was a score of $1.3 \pm 0.3$. However, CPI-1189 at $10 \mathrm{mg} \mathrm{kg}^{-1}$ p.o. had no inhibitory effect in this series of experiments on the TNBS-induced increase in MPO or tissue wet weight. 


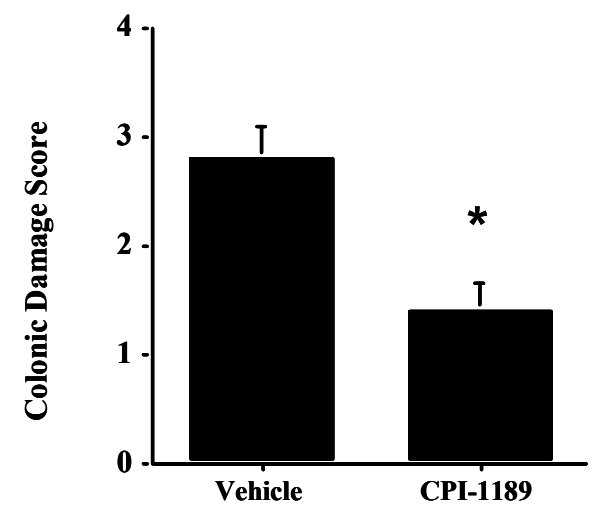

(a)

(b)
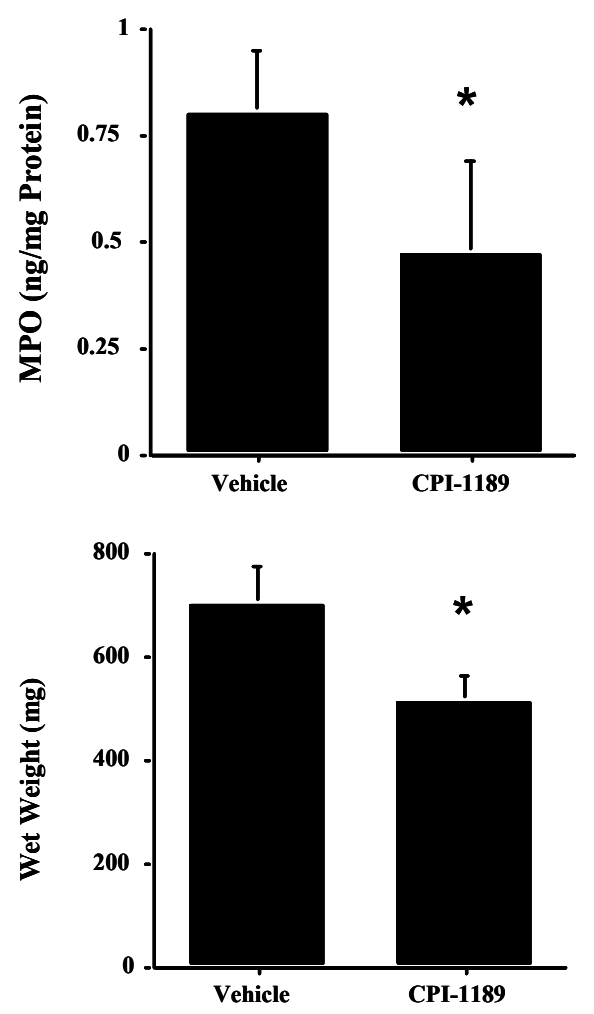

(c)

Fig. 2: Effect of CPI-1189 on the TNBS induced colonic inflammation. Rats $(n=14$ /group) were administered by oral gavage a single dose of CPI-1189 (or the methylcellulose vehicle) at a dose of $30 \mathrm{mg} \mathrm{kg}^{-1} 1 \mathrm{hr}$ before the TNBS enema. Three days post TNBS the rats treated with CPI-1189 showed a reduction in the colonic damage score (A), a decrease in the MPO activity (B) and a decrease in the wet weight of the colon (C) compared to control rats that were treated with the methylcellulose vehicle. Values are mean \pm sem. * Statistically significant different from controls $\mathrm{p}<0.05$

In a second series of experiments, groups of rats ( $n=14$ /group) were treated with either a single oral dose of CPI-1189 (30 $\left.\mathrm{mg} \mathrm{kg}^{-1}\right)$ or the methylcellulose vehicle control $1 \mathrm{hr}$. before the TNBS enema. Macroscopic analysis after 3 days revealed that CPI1189 provided

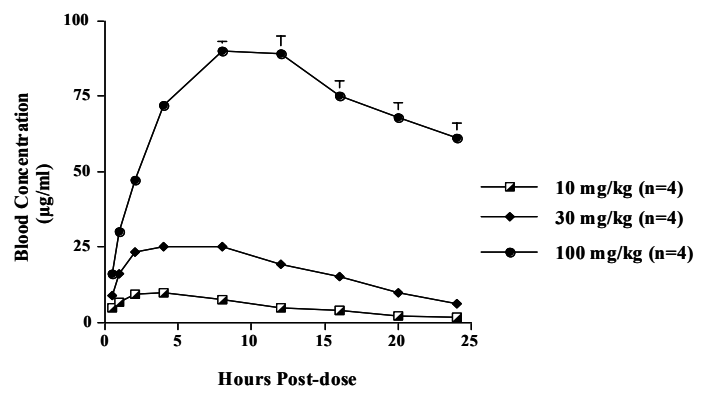

\begin{tabular}{|c|c|c|c|c|c|}
\hline \multicolumn{6}{|c|}{$\begin{array}{l}\text { Mean Pharmacokinetic Parameter Estimates O SE Following po Adminis tration } \\
\text { of } 3 \text { Ascending Doses of CPI-1189 to Rats. }\end{array}$} \\
\hline $\begin{array}{l}\text { Dose } \\
(\mathrm{mg} / \mathrm{kg})\end{array}$ & $\frac{T_{\max }}{(h)}$ & $\begin{array}{l}C_{\text {gar }} \\
(E g / m L)\end{array}$ & $\begin{array}{l}A U C_{0 \rightarrow 24 h} \\
(\hat{E} g \times h r / m L)\end{array}$ & $\begin{array}{l}t_{1 / 2} \\
h)\end{array}$ & $\begin{array}{ll}T / F \\
(L / k g)\end{array}$ \\
\hline 10 & $3.5 \mathrm{O} 0.5$ & 9.600 .3 & 12305.8 & 7.401 .2 & 0.7700 .13 \\
\hline 30 & 5.501 .5 & 2601.3 & $413 \bigcirc 28$ & 5.900 .7 & $0.54 \bigcirc 0.03$ \\
\hline 100 & 1001.2 & 9303.6 & 1731077 & $\begin{array}{l}\text { Not } \\
\text { Determined }\end{array}$ & Not Determined \\
\hline
\end{tabular}

Fig. 3: Plot of mean blood concentrations $\left(\mu \mathrm{g} \mathrm{mL}^{-1} \pm \mathrm{SD}\right)$ and a table of resulting mean pharmacokinetic parameter estimates ( \pm SD) following p.o. administration of three ascending doses of CPI-1189 to rats ( $n=4 /$ dose group). Circles, diamond and squares in the plot are data from the 10,30 and $100 \mathrm{mg} \mathrm{kg} \mathrm{kg}^{-1}$ treatments, respectively. Approximately proportional increases in $\mathrm{C}_{\max }$ and $\mathrm{AUC}$ are observed as the dose level is increased from 10 to $100 \mathrm{mg} \mathrm{kg}$. Similar shapes and $T_{\max }$ values for the blood concentration-time profiles are observed for the 10 and $30 \mathrm{mg} \mathrm{kg}^{-1}$ dose group. However, the profile for the $100 \mathrm{mg} \mathrm{kg}^{-1}$ dose group shows evidence of rate- limited absorption, i.e.,increased Tax and diminished fall off of blood concentrations at later time points

significant protection against the severe inflammatory response of the colonic mucosa induced by TNBS (Fig. $2 \mathrm{~A})$. In these same animals we also determined the effect of CPI-1189 on TNBS-induced increases in MPO activity and tissue wet weight. Our findings showed that CPI-1189 (30 mg kg-1 p.o.) produced a significant reduction in MPO activity (Fig. 2B) and tissue wet weight (Fig. 2C) compared to vehicle-treated animals.

Lack of a protective effect with PBN: In this series of experiments, the efficacy of PBN against TNBSinduced colitis was investigated. Due to the acid instability of PBN, the compound was given via the i.p. rather than the p.o. route of administration. Groups of rats ( $\mathrm{n}=6$ /group) were treated with a single dose of PBN (150 mg kg ${ }^{-1}$ i.p.) or the saline vehicle control i.p. $1 \mathrm{hr}$. before the TNBS enema. Macroscopic analysis of the colon after 3 days revealed that PBN did not protect 
against the severe inflammatory response of the colonic mucosa induced by TNBS. In the PBN treated group the mean morphological score of $2.4 \pm 1.0$ did not differ significantly from the saline vehicle-treated group where the morphological score was $2.6 \pm 0.9$.

Evaluation of dose proportionality studies: Significant blood concentrations of CPI-1189 could be measured for

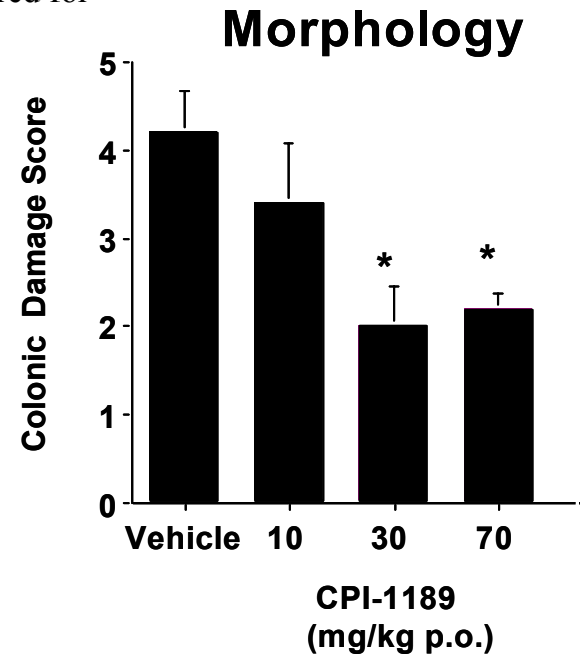

(a)

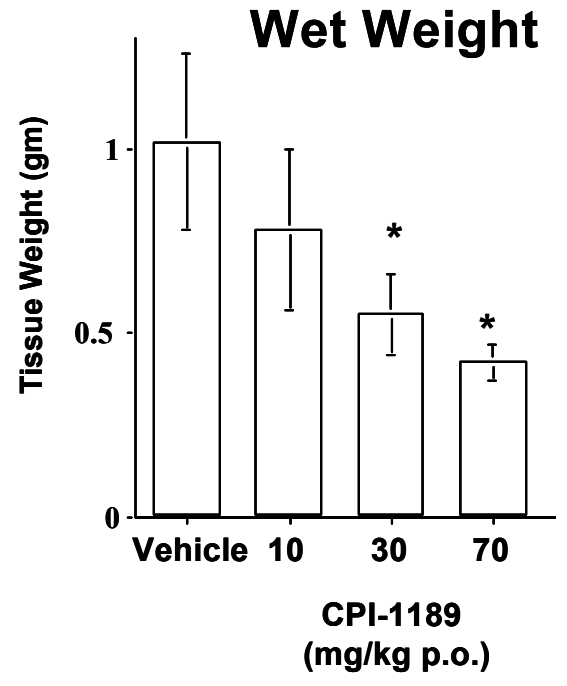

(b)

Fig. 4: Dose-response effect of CPI-1189 (10, 30 or $70 \mathrm{mg} \mathrm{kg}^{-1}$ p.o.) or the methylcellulose vehicle on TNBS $\left(50 \mathrm{mg} \mathrm{kg}^{-1}\right)$ induced colonic inflammation. Rats $(\mathrm{n}=5$ /group) were dosed $1 \mathrm{hr}$ before the TNBS enema. At the $10 \mathrm{mg} \mathrm{kg}^{-1}$ dose of CPI-1189 there was a reduction in the colonic damage score which was not statistically different from the vehicletreated control rats. CPI-1189 at doses of 30 and $70 \mathrm{mg} \mathrm{kg}^{-1}$ significantly reduced the TNBS colonic damage score. Values are mean $\pm \mathrm{sem}$. Statistically significant difference at $\mathrm{p}<0.05$ up to $24 \mathrm{hr}$. post p.o. doses of 10,30 and $100 \mathrm{mg} \mathrm{kg}^{-1}$ (Fig. 3). Resulting pharmacokinetic parameter estimates show excellent dose proportionality for $\mathrm{C}_{\max }$ and AUC values. However, $\mathrm{T}_{\max }$ increased from 3.5 to $10 \mathrm{hr}$. as the dose was increased from 10 to $100 \mathrm{mg} \mathrm{kg}^{-1}$, suggesting that at higher doses absorption of the drug was rate limited but with a similar extent of absorption as achieved at lower doses. For the $100 \mathrm{mg} \mathrm{kg}^{-1}$ dose, an insignificant fall off of blood concentrations over the $24 \mathrm{hr}$. sampling period prevented a calculation of $t_{1 / 2}$ and $\mathrm{V}_{\mathrm{z}} / \mathrm{F}$ values and probably produced an under estimation

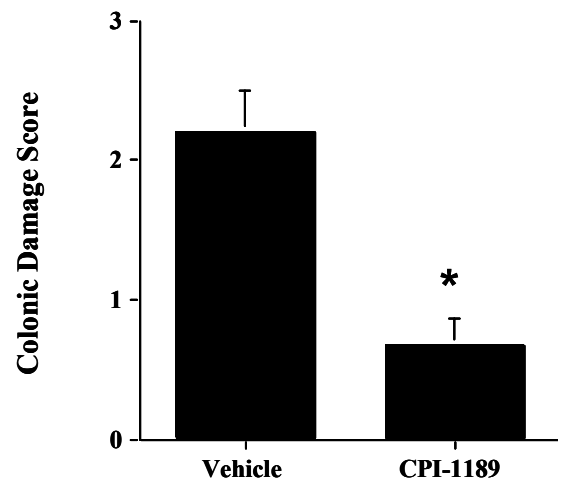

(a)

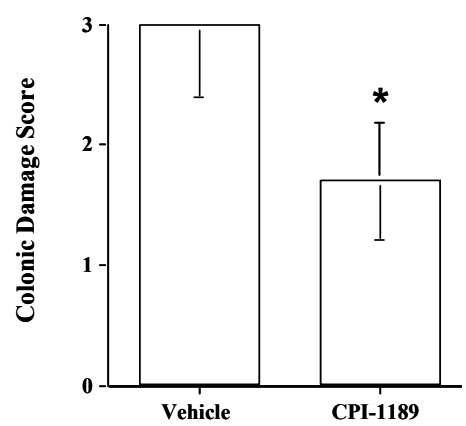

(b)

Fig. 5: Effect of route of administration on CPI-1189 (30 mg kg$\left.{ }^{-1}\right)$ on the TNBS induced colonic inflammation. Rats $(\mathrm{n}=$ 6/group) were dosed either p.o. or i.v. $1 \mathrm{hr}$. before the TNBS enema or the vehicle (1\% aqueous methylcellulose for p.o. and $25 \%$ aqueous PEG for i.v.). Although both routes of administration of CPI-1189 were protective against TNBSinduced damage, there was a more marked reduction in the colonic damage score in rats given the CPI-1189 via the i.v. route (A) compared to rats predosed with CPI-1189 p.o. (B). Values are mean \pm sem. * Statistically significant difference at $\mathrm{p}<0.05$

of AUC for this dose level. For the 10 and $30 \mathrm{mg} \mathrm{kg}^{-1}$ doses, the $\mathrm{t}_{1 / 2}$ and $\mathrm{V}_{\mathrm{z}} / \mathrm{F}$ values were approximately $6 \mathrm{hr}$. and $0.6 \mu \mathrm{L} \mathrm{kg}^{-1}$, respectively.

Evaluation of the dose-response characteristics of CPI-1189: The objective of this series of experiments was to determine the dose-response characteristics of 
CPI-1189 given orally in the TNBS rat model of colitis. Groups of rats $(n=5 /$ group) were administered a single oral dose of CPI-1189 (10-70 mg kg-1) given $1 \mathrm{hr}$. before the TNBS. In the vehicle-treated rats, the TNBS enema stimulated gross morphological damage in the colon and increased the wet weight of the tissue (Fig. 4). In rats pretreated with CPI-1189 there was a significant and a dose-dependent reduction in both the TNBS-induced colonic damage (Fig. 4A) and increase in tissue wet weight (Fig. 4B). MPO levels were not assessed during this series of experiments.
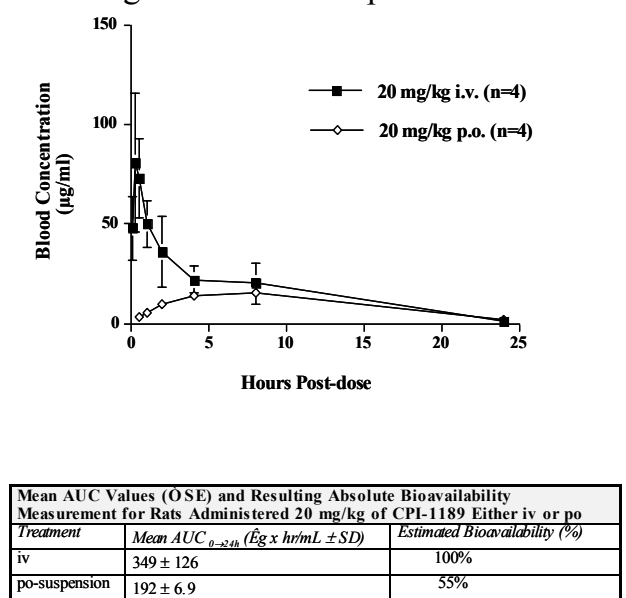

Fig. 6: Plot of mean blood concentrations $\left(\mu \mathrm{g} \mathrm{mL}^{-1} \pm \mathrm{SD}\right)$ and a table of mean AUC values $( \pm \mathrm{SD}$ ) following either i.v. or p.o. administration of a single $20 \mathrm{mg} \mathrm{kg}{ }^{-1}$ dose of CPI-1189 to rats ( $n=4 /$ dose group). Circles and squares in the plot are data from the p.o. and iv. treatments, respectively. The mean value for the terminal elimination half life observed following either treatments is similar, approximately $5 \mathrm{hr}$. This mean value is also similar to mean value for the terminal elimination half life found in the dose proportionality study (Fig. 3). Based on AUC values, the systemic bioavailability of a the p.o. dose of CPI-1189 is approximately half the systemic bioavailability of an i.v. dose
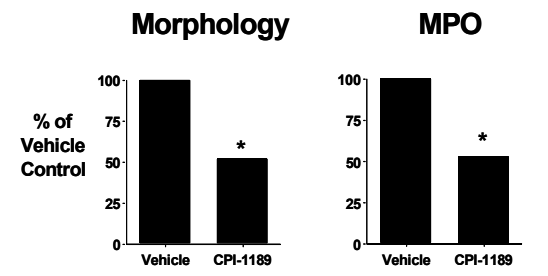

\section{Wet Weight}

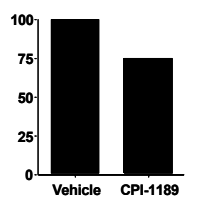

Fig. 7: Effect of CPI-1189 (30 mg kg$\left.{ }^{-1}\right)$ in the TNBS-induced rodent model of colitis when dosed via oral gavage at 4,8 and $24 \mathrm{hr}$ after the TNBS challenge $(\mathrm{n}=14)$. Control rats received 3 doses of the methylcellulose vehicle at 4,8 and 24 $\mathrm{hr}$ after the TNBS challenge $(\mathrm{n}=14)$. Following these 3 doses of CPI-1189 (cumulative dose of $90 \mathrm{mg} \mathrm{kg}^{-1}$ ) there was a significant reduction in the TNBS colonic damage score compared to the vehicle control rats. Values are mean \pm sem. * Statistically significant difference at $\mathrm{p}<0.05$

These results suggest that oral administration of CPI1189 induces significant protective effects against
TNBS-induced colonic damage at doses between of 30 and $70 \mathrm{mg} \mathrm{kg}^{-1}$. As there was little difference between the protective effects of 30 and $70 \mathrm{mg} \mathrm{kg}^{-1}$ an additional experiment was performed with a higher dose of CPI-1189 (90 mg kg-1) to confirm that the maximum protective effect had been reached. In these experiments we found no obvious enhancement of

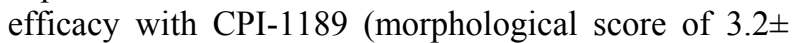
0.5 in the vehicle-treated group and $2.0 \pm 0.4$ in the CPI-1189 group, a protection of $37.5 \%$.

Influence of route of administration: In the next series of experiments, we compared the efficacy of a single dose of CPI-1189 (30 $\left.\mathrm{mg} \mathrm{kg}^{-1}\right)$ administered by two defined routes: intravenously or orally. CPI-1189 was administered $1 \mathrm{hr}$. before the TNBS enema. Rats ( $n=6 /$ dosing group) pretreated with the appropriate vehicle, p.o. (1\% aqueous methylcellulose) or i.v. (25\% aqueous PEG) $1 \mathrm{hr}$. before the TNBS served as controls. For the same $30 \mathrm{mg} \mathrm{kg}^{-1}$ dose, greater morphological protection $(77 \%)$ was observed when CPI-1189 was administered intravenously (Fig. 5A) compared to rats in which the CPI-1189 was administered p.o. (Fig. 5B).

Evaluation of absolute bioavailability: Significant blood concentrations of CPI-1189 could be measured for up to $24 \mathrm{hr}$. post administration of $20 \mathrm{mg} \mathrm{kg}^{-1}$ i.v. or p.o. doses of CPI-1189 (Fig. 6). Resulting AUC and $\mathrm{C}_{\max }$ values following the p.o. dose (Fig. 6) are consistent with those found in the dose proportionality study. The AUC values following the i.v. doses were higher than the p.o. doses, leading to a calculated absolute bioavailability of approximately 55\%. Insufficient blood sampling in the 8-24 hr. post-dose time period for the animals treated p.o. limits the validity of parameter estimates $\pm S D$ for the $t_{1 / 2}$ and $\mathrm{V}_{\mathrm{z}} / \mathrm{F}$ values obtained. Nevertheless, the mean values for these parameters \pm SD following p.o. dosing, $5.4 \pm 0.6$ $\mathrm{hr}$. and $0.75 \mu \mathrm{L} \mathrm{kg}^{-1}$, respectively, are similar to those found in the dose proportionality study. In addition, the $\mathrm{t}_{1 / 2}$ values $\pm \mathrm{SD}$ found following the i.v. administration, $4.8 \pm 0.8 \mathrm{hr}$. are also very similar to that found after oral administration in the absolute bioavailability study and the dose proportionality study.

Healing effects of CPI-1189 during acute posttreatment: In this series of experiments we investigated the effects of CPI-1189 administered after the TNBS enema to determine the effectiveness of the compound during the acute phase of the TNBS-induced inflammatory response. CPI-1189 (90 $\mathrm{mg} \mathrm{kg}^{-1}$ p.o) 
administered between 4,8 or $24 \mathrm{hr}$ after the TNBS enema reduced the colonic damage induced by TNBS (Fig. 7).

\section{DISCUSSION}

In the present study TNBS in ethanol induced macroscopic damage of the distal colon in rats ${ }^{[6,8]}$ and stimulated an increase in the activity of MPO, an enzymatic marker of neutrophil infiltration into damaged tissue ${ }^{[9,10]}$. It was also found that CPI-1189, a novel chemical entity, was protective in this model of colonic damage. CPI-1189's protective effects could be observed on tissue appearance; through measures of morphology and colonic wet weights and on tissue biochemistry through the measurement of MPO activity. Morphology damage scores, tissue wet weights and MPO values increased significantly in TNBS animals pretreated with vehicle, whereas in TNBS animals treated with CPI-1189 there was a significant reduction in these measures, suggesting that CPI-1189 produced an anti-inflammatory effect. The protection afforded by oral dosing of CPI-1189 was modest and inconsistent at a dose of $10 \mathrm{mg} \mathrm{kg}^{-1}$ or less, but statistically significant at $30 \mathrm{mg} \mathrm{kg}^{-1}$ or greater.

CPI-1189 was also effective when administered intravenously suggesting that the protection is affected by systemic, not topical action. The greatest degree of protection, $77 \%$, was seen when CPI-1189 was administered at $30 \mathrm{mg} \mathrm{kg}^{-1}$ i.v., 1 hour before the TNBS enema. This level of protection is $50 \%$ greater than when a $30 \mathrm{mg} \mathrm{kg}^{-1}$ dose of CPI-1189 is given p.o. and is consistent with the approximately $50 \%$ absolute bioavailability of CPI-1189 in rat. Although a $70 \mathrm{mg}$ $\mathrm{kg}^{-1}$ p.o. dose of CPI-1189 caused a further improvement in the TNBS-induced increase in wet weight of the tissue beyond that seen with the $30 \mathrm{mg}$ $\mathrm{kg}^{-1}$ dose, this dose of CPI-1189 did not further improve the morphologic score beyond that seen with $30 \mathrm{mg} \mathrm{kg}^{-1}$ CPI-1189. In a separate experiment, these findings with the 30 and $70 \mathrm{mg} \mathrm{kg}^{-1}$ doses were extended to treatment with a $90 \mathrm{mg} \mathrm{kg}^{-1}$ dose of CPI1189. In this experiment, protection based on morphologic score was little different than that found following the 30 or $70 \mathrm{mg} \mathrm{kg}^{-1}$ treatment with CPI1189 , but the protection based on wet weight measurements (edema) was better than that observed following the 30 or $70 \mathrm{mg} \mathrm{kg}^{-1}$ treatment with CPI1189. Based upon the proportional increase in systemic exposure (AUC values) when the CPI-1189 p.o. dose is increased from 10 to $100 \mathrm{mg} \mathrm{kg}^{-1}$, an improvement in the morphologic score with the higher dose of CPI-
1189 would be expected. However, a consideration of the blood concentrations present in the rat when the TNBS treatment is administered gives a possible explanation for these findings and also points to the possible need for CPI-1189 to be present in blood at a reasonably high concentration at the time of TNBS instillation to affect the greatest protection. The absorption of CPI-1189 at doses above $30 \mathrm{mg} \mathrm{kg}^{-1}$ appears to be rate limited, the blood concentrations one hour post-dose (the time of TNBS enema) for the 30 , 70 and $90 \mathrm{mg} \mathrm{kg}^{-1}$ doses are not significantly different (approximately 16, 23 and $28 \mu \mathrm{g} \mathrm{mL}^{-1}$, respectively. At this same time point the blood concentration following a $10 \mathrm{mg} \mathrm{kg}^{-1}$ p.o. dose is approximately one third the concentrations achieved following the $30 \mathrm{mg} \mathrm{kg}^{-1}$ dose which is consistent with the lower and more inconsistent activity observed at this dose. When the degree of protection produced following i.v. administration of CPI-1189 is considered, it is apparent that blood concentrations of the compound are approximately three times greater than those seen following oral administration of the same dose. These higher blood concentrations can readily account for the higher degree of protection observed with i.v. dosing. These finding suggest the need for CPI-1189 to be present in blood at a relatively high concentration at the time of the TNBS instillation to produce the greatest protection. Despite the apparent need for CPI-1189 to be present for the best effect at relatively high concentrations in the blood at the time of the TNBS insult, the compound also appears to have some activity when given post-TNBS. In a preliminary study to evaluate the protective effects of CPI-1189 when given after the TNBS enema, the efficacy of a multiple dosing regime of orally administered CPI-1189 was examined. The degree of protection observed was comparable to that seen when CPI-1189 was given $1 \mathrm{hr}$. prior TNBS administration. The mechanism for this post-TNBS protection may be more related to the previously mentioned highly dose dependent decrease in edema found with CPI-1189 than the mechanism responsible for protection based on morphologic score which seems to plateau at $30 \mathrm{mg} \mathrm{kg}^{-1}$. However, these findings, taken together with previous results, show that CPI1189 not only protects the colonic tissue against an inflammatory insult but that the compound may actually interrupt or attenuate in the inflammatory process even after the process has been initiated. Although this hypothesis requires further experimental investigation, these findings of anti-inflammatory activity of CPI-1189 suggest that the compound could have clinical applications against both an active 
inflammatory response and as prophylaxis against a new inflammatory insult. The finding that CPI-1189 is efficacious against TNBS-induced colitis is also in agreement with findings in another in vivo inflammatory model, that of adjuvant induced arthritis model of chronic inflammation, in which CPI-1198 was able to modulate the inflammatory response (data on file, Centaur Pharmaceuticals, Inc.).

The mechanism by which CPI-1189 protects in the TNBS model is still not fully understood and remains under active investigation. CPI-1189 has comparable radical trapping activity to $\mathrm{PBN}$ and has also been shown to attenuate TNF- $\alpha$ induced production of reactive oxygen species, however PBN shows little efficacy compared to CPI-1189 in the TNBS-induced model of colitis. This suggests that a free radical trapping mechanism is not responsible for the efficacy seen in this model. One possibility is that CPI-1189 modulates the activity of TNF- $\alpha$ or IL-1 $\beta$, two cytokines thought to be involved in the TNBS induced damage while PBN does not.

Both of these cytokines are elevated following TNBS administration ${ }^{[11-14]}$ and treatment with an IL-1 receptor antagonist ${ }^{[15,16]}$ and antibodies against TNF- $\alpha$ ${ }^{[13]}$ reportedly ameliorate the effect of TNBS in rodents. Consistent with this possibility, CPI-1189 was recently shown to prevent TNF- $\alpha$ induced apoptosis in vitro ${ }^{[17,18]}$ to protect against TNF- $\alpha$ induced weight loss, cognitive deficits and cell death in vivo ${ }^{[19,20]}$ and to prevent the induction of stress activated protein kinases (p38MAPK) by IL-1 $\beta$ in vitro ${ }^{[21]}$. Alternatively the protective effects of CPI-1189 against colonic damage in this rat model may arise from its ability to alter the cascade of oxidative events associated with TNBS treatment ${ }^{[22,23]}$. CPI-1189 has activity as a scavenger of hydroxyl radical similar to that of PBN (data not shown) and the compound has been shown to reduce the generation of ROS attendant to TNF- $\alpha$ treatment ${ }^{[17]}$. The likelihood that CPI-1189 protects against TNBS-induced damage by specifically targeting cytokine-mediated damage has considerable relevance to IBD. Both of these cytokines are elevated in IBD patients and current thinking implicates both TNF- $\alpha$ and IL-1 as important therapeutic targets for both Crohn's disease and ulcerative colitis ${ }^{[5]}$. Indeed the emergance of genetically engineered antibodies that bind TNF. $\alpha$ (eg. inflixamab) have recently been approved for patient use. In summary, the present findings suggest that small molecules, such as CPI1189, that also target these pathways and possess protective and healing activity in animal models of IBD following oral administration might provide similar efficacy in the human disease.

\section{ACKNOWLEDGMENTS}

The work was supported by grants from Centaur Pharmaceuticals Inc. and the NIH-SBIR program Grant \# DK 1R43DK51902-01. The authors would like to thank Dr. Kalina Venkova for conducting the MPO assays and Dr. Larry Peters for the morphological scoring of the colonic samples.

\section{REFERENCES}

1. Levin, B., 1992. Inflammatory bowel disease and colon cancer. Cancer, 70: 1313-1316.

2. Calkins, B.M. and AI. Mendeloff, 1986. Epidemiology of inflammatory bowel disease. Epidemiol. Rev., 8: 60-91.

3. Novelli, G.P., 1992. Oxygen radicals in experimental shock: Effects of spin trapping nitrones in ameliorating shock pathophysiology. Crit. Care Med., 20: 499-507.

4. Hensley, K., J.M. Carney, C.A. Stewart, T. Tabatabaie, Q. Pye and R.A. Floyd, 1997. Nitronebased free radical traps as neuroprotective agents in cerebral ischaemia and other pathologies. Int. Rev. Neurobiol., 40: 299-317.

5. Papadakis, KA. and S.R. Targen, 1999. Current theories on the causes of inflammatory bowel disease. Gastroenterol. Clin. North Am., 28: 283-96.

6. Morris, G.P., P.L. Beck, M.S. Herridge, W.T. Depew, M.R. Szewczuk and J.L. Wallace, 1989. Hapten-induced model of chronic inflammation and ulceration in the rat colon. Gastroenterology, 96: 795-803.

7. Krawisz, J.E., P. Sharon and W.F. Stenson, 1984. Quantitative assay for acute intestinal inflammation based upon myeloperoxidase activity. Assessment of inflammation in rat and hamster models. Gastroenterology, 87: 1344-50.

8. Boughton-Smith, N.K., J.L.Wallace, G.P. Morris and B.J. Whittle, 1988. The effect of antiinflammatory drugs on eicosanoid formation in a chronic model of inflammatory bowel disease in the rat. Br. J. Pharmacol., 94: 65-72.

9. Castro, G.A., S.A. Roy and R.D. Stockstill, 1974. Trichinella spiralis: peroxidase activity in isolated cells from the rat intestine. Exp Parasitol., 36: 307315. 
10. Smith, J.W. and G.A. Castro, 1978. Relation of peroxidase activity in gut mucosa to inflammation. Am. J. Physiol., 234: R72-R79.

11. McHugh, K.J., S.M. Collins and H.P. Weingarten, 1994. Central interleukin-1 receptors contribute to suppression of feeding after acute colitis in the rat. Am. J. Physiol., 266: R1659-63.

12. Ameho, C.K., A.A. Adjei, E.K. Harrison, K. Takeshita, T. Morioka, Y. Arakaki, E. Ito, I. Suzuki, AD. Kulkarni, A. Kawajiri and S. Yamamoto, 1997. Prophylactic effect of dietary glutamine supplementation on interleukin 8 and tumor necrosis factor alpha production in trinitrobenzene suphonic acid induced colitis. Gut, 41: 487-93.

13. Jacobson, K., K. McHugh, S.M. Collins, 1997. The mechanism of altered neural function in a rat model of acute colitis. Gastroenterology, 112: 15662.

14. Videla, S., A. Garcia-Lafuente, M. Antolin, J. Vilaseca, F. Guarner, E. Crespo, G. Gonzalez, A. Salas and J.R. Malagelada, 1998. Anti-tumor necrosis factor therapy in rat chronic granulomatous colitis: Critical dose-timing effects on outcome. J. Pharmacol. Exp. Ther., 287: 854-9.

15. McCafferty, D.M., K.J. Rious and J.L. Wallace, 1992. Granulocyte infiltration in experimental colitis in the rat is interleukin-1 dependent and leukotriene independent. Eicosanoids, 5: 121-5.

16. Tateishi, H., K. Mitsuyama, A. Toyonaga, M. Tomoyose and K. Tanikawa, 1997. Role of cytokines in experimental colitis: Relations to intestinal permeability. Digestion, 58: 271-81.

17. Irwin, I., E. MacGowan, W.D. Flitter and W.A. Garland, 1998. CPI-1189 attenuates the toxic effects of TNF- $\alpha$ in SK-N-MC cells. Abs. for Society of Neuroscience, 2: 596.8 .
18. Pulliam, I., M. Zhou M. Stubblebine and CM. Bitler, 1998. Differential modulation of cell death proteins in human brain cells by tumor necrosis factor alpha and platelet activating factor. $\mathrm{J}$. Neurosci. Res., 54: 530-8.

19. Bjugstad, K.B., W.D. Flitter, W.A. Garland, G.C. $\mathrm{Su}$ and G.W. Arendash, 1998. Preventive actions of a synthetic antioxidant in a novel animal model of AIDS dementia. Brain Res., 795: 349-57.

20. Bjugstad, K.B., W.D. Flitter, W.A. Garland R.M. Philpot, C.L., Kirstein, G.W. Arendash, 2000. CPI1189 prevents apoptosis and reduces glial fibrillary acidic protein immunostaining in a TNF alpha infudion model for AIDs dementia complex. J. Neurovirol., 6: 478-491.

21. Hensley, K., K.A. Robinson, Q.N. Pye, R.A. Floyd, I. Cheng, W.A. Garland and I. Irwin, 2000. CPI-1189 inhibits IL-1 $\beta$-induced p38-MAP kinase phosphorylation: an explanation for its neuroprotective properties. Neuroscience Lett., 10: 281: 179-182.

22. Grisham, M.B., C. Volkmer, P. Tso and T. Yamada, 1991. Metabolism of trinitrobenzene sulphonic acid by the rat colon produces reactive oxygen species. Gastroenterology, 101: 540-7.

23. Zea-Iriarte, W.L., K. Makiyama, S. Goto, K. Murase, Y. Urata, I. Sekine, K. Hara and T. Kondo, 1996. Impairment of antioxidants in colonic epithelial cells isolated from trinitrobenzene sulphonic acid-induced colitis rats: Protective effects of rebamipide. Scand. J. Gastroenterol., 31: 985-992. 